\title{
Lenvatinib-Induced Tumor-Related Hemorrhages in Patients with Large Hepatocellular Carcinomas
}

Uchida-Kobayashi S. Kageyama K. Yamamoto A. Ikenaga H. Yoshida K. Kotani K. Kimura K. Odagiri N. Hagihara A. Fujii H. Enomoto M. Tamori A. Kubo S. Miki Y. Kawada N.

\begin{tabular}{|c|l|}
\hline Citation & Oncology. 99; $186-191$. \\
\hline Issue Date & $2021-03$ \\
\hline Published & $2020-10-08$ \\
\hline Type & Journal Article \\
\hline Textversion & author \\
\hline Relation & $\begin{array}{l}\text { This is the peer-reviewed but unedited manuscript version of the following article: } \\
\text { Oncology } 2021 ; 99: 186-191 \text { (DOI: } 10.1159 / 000510911) . \\
\text { The final, published version is available at } \text { https://doi.org/10.1159/000510911. }\end{array}$ \\
\hline DOI & $10.1159 / 000510911$ \\
\hline
\end{tabular}

Self-Archiving by Author(s)

Placed on: Osaka City University Repository

Uchida-Kobayashi, S., Kageyama, K., Yamamoto, A., Ikenaga, H., Yoshida, K., Kotani, K., Kimura, K., Odagiri, N., Hagihara, A., Fujii, H., Enomoto, M., Tamori, A., Kubo, S., Miki, Y., \& Kawada, N. (2020). Lenvatinib-Induced Tumor-Related Hemorrhages in Patients with Large Hepatocellular Carcinomas. Oncology, 1-6. https://doi.org/10.1159/000510911 


\section{Research Article \\ Manuscript Title}

\section{Lenvatinib-induced tumor-related hemorrhages in patients with large hepatocellular carcinomas}

Sawako Uchida-Kobayashi ${ }^{\mathrm{a}}$, Ken Kageyama ${ }^{\mathrm{b}}$, Akira Yamamoto $^{\mathrm{b}}$, Hiroko Ikenaga ${ }^{\mathrm{a}}$, Kanako Yoshida ${ }^{\mathrm{a}}$, Kohei Kotani ${ }^{\mathrm{a}}$, Kenjiro Kimura ${ }^{\mathrm{c}}$, Naoshi Odagiria ${ }^{\mathrm{a}}$, Atsushi Hagihara ${ }^{\mathrm{a}}$ Hideki Fujii $^{\mathrm{a}}$, Masaru Enomoto ${ }^{\mathrm{a}}$, Akihiro Tamori $^{\mathrm{a}}$, Shoji Kubo ${ }^{\mathrm{c}}$, Yukio Miki ${ }^{\mathrm{b}}$, Norifumi Kawada ${ }^{\mathrm{a}}$

a Department of Hepatology, Graduate School of Medicine, Osaka City University, Osaka, Japan

${ }^{\mathrm{b}}$ Department of Diagnostic and Interventional Radiology, Graduate School of Medicine, Osaka City University, Osaka, Japan

${ }^{c}$ Department of Hepato-Biliary-Pancreatic Surgery, Graduate School of Medicine, Osaka City University, Osaka, Japan

Short Title: Vascular lake phenomenon in lenvatinib treatment

Corresponding Author:

Sawako Uchida-Kobayashi, MD \& PhD

Department of Hepatology

Graduate School of Medicine, Osaka City University, Osaka, Japan

1-4-3, Asahi-machi, Abenoku,

Osaka 545-8585, Japan.

Tel: $+81-6-6645-3905$

E-mail: sawako@med.osaka-cu.ac.jp 
Number of Tables: 3

Number of Figures: 1

Word count: 1825 words

Keywords: hepatocellular carcinoma, hemorrhage, lenvatinib, vascular lake phenomenon, tyrosine-kinase inhibitor 


\section{Abstract}

Introduction: Lenvatinib has been approved as a systemic therapy for patients with unresectable hepatocellular carcinoma (HCC). We recently experienced lenvatinib-induced tumor-related hemorrhage in patients with HCC. The full details of tumor-related hemorrhage as a lenvatinib-related adverse event have not been elucidated.

Methods: This was a retrospective single-center study that enrolled consecutive patients treated with lenvatinib for unresectable HCC from April 2018 to February 2020.

Results: Sixty-eight consecutive patients were enrolled in this study. Among them, 5 cases developed intraperitoneal or intratumoral hemorrhages. The patients with hemorrhage had larger tumors (maximum tumor size; $97.5 \pm 46.4 \mathrm{~mm}$ and $38.2 \pm 28.8 \mathrm{~mm}$, respectively. $\mathrm{p}=0.009$ ) than the patients without hemorrhage. The dosing period of lenvatinib (median, 3 and 93 days, respectively. $p<0.001$ ) and the survival time from initial administration of lenvatinib (median, 77 and 495 days, respectively. $\mathrm{p}<0.001)$ of the patients with hemorrhage were shorter than those of the patients without hemorrhage. Especially, in four cases with large HCCs (maximum tumor diameter was $>90 \mathrm{~mm}$ ), tumor hemorrhage with vascular lake-like phenomenon were evident, although most tumor blood flow was suppressed.

Discussion/Conclusion: It becomes clear that lenvatinib treatment brings about tumor-related hemorrhages despite rapid suppression of tumor blood flow. We speculate that lenvatinib quickly blocks the feeding circulation resulting in the tumor hemorrhage by necrosis. Clinicians should pay careful attention to the development of life-threatening hemorrhages when treating large HCCs with lenvatinib. 


\section{Introduction}

Primary liver cancer is the seventh most frequently occurring cancer and the second most common cause of cancer mortality in the world. Hepatocellular carcinoma (HCC) is the dominant type of liver cancer, accounting for approximately $75 \%$ of the total [1-3].

Molecular-targeted agents (MTA) therapy is the guideline-recommended global standard of care for patients with well-preserved liver function (Child-Pugh A) and with advanced tumors, Barcelona Clinic Liver Cancer stage (BCLC)-C, or earlier stage tumors progressing upon or unsuitable for locoregional therapies [4]. In Japan, lenvatinib is available as a first-line MTA. Lenvatinib is an orally active tyrosine kinase inhibitor, and it exerts antitumor effects by suppressing tumoral blood flow. The survival benefits of lenvatinib were demonstrated in phase 3 clinical trials, the REFLECT trial, involving patients with unresectable HCC [5]. In addition, some recent real-world, retrospective, multicenter studies reported high efficacy and tolerability of Lenvatinib in the patients with unresectable HCC $[6,7]$.

Recently, we reported two cases of HCCs in which tumor related-hemorrhages were caused by lenvatinib [8]. To the best our knowledge, no previous report on HCC has identified tumor-related hemorrhage as an MTA-related adverse event (AE) other than our case reports, and the full details of this tumor hemorrhage effect have not been elucidated.

In this study, we examined tumor related-hemorrhages in patients treated with lenvatinib for unresectable HCC.

\section{Materials and Methods}

\section{Patients}

This was a retrospective single-center study that enrolled consecutive patients administered lenvatinib for unresectable HCCs between April 2018 and February 2020 in Osaka City University Hospital. The diagnostic criteria for HCC, which have been described previously [9], include hyperattenuation during the arterial phase or hypoattenuation during the portal phase, as revealed by dynamic computed tomography (CT) or magnetic resonance imaging. We reviewed the clinical backgrounds, radiological findings, clinical courses, and AEs of the patients who were given lenvatinib for HCCs. This study was conducted according to the guidelines of the Declaration of Helsinki. Written informed consent was obtained from all patients prior to treatment. The study protocol was approved by the Ethics Committee of Osaka City University Hospital (No. 3720).

\section{Treatment}


Lenvatinib were administered orally dependently to the patient's weight; those who weighed $<60 \mathrm{~kg}$ or $\geq 60 \mathrm{~kg}$ were initially given 8 or $12 \mathrm{mg}$ lenvatinib daily, respectively. A dose reduction and interruptions of Lenvatinib, depending on the general condition and AEs, were permitted.

\section{Statistical Analyses}

Statistical analysis was performed with EZR software (ver. 1.37; Saitama Medical Center, Jichi Medical University, Saitama, Japan). Data are expressed as means \pm standard deviation, medians with ranges (25-75th percentiles), or numbers. Statistical analyses were performed using the Wilcoxon signed-rank test, the chi-square test and the Kaplan-Meier method. Values of $\mathrm{P}<0.05$ were considered statistically significant.

\section{Results}

\section{Patient Characteristics}

We prescribed lenvatinib for 87 patients with unresectable HCCs from April 2018 to February 2020. Among them, 68 consecutive patients with measurable lesions in the liver were enrolled in this study. Baseline patient characteristics are shown in Table 1. The median patient age was 72 (55-78) years. Fifty-nine (86.8\%) patients were male, and 26 (38.2\%) had BCLC-C HCCs. Sixty-three (92.7\%) were clinically in Child-Pugh class A. Six $(8.8 \%)$ had no history of previous HCC treatment. The average maximum tumor diameter was $42.6 \pm 33.7 \mathrm{~mm}$.

\section{Clinical course}

Initiation doses of lenvatinib were 12,8 or $4 \mathrm{mg} /$ day, and $51(75 \%)$ patients started taking the standard dose. Among them, five patients (7.4\%) developed intraperitoneal or intratumoral bleeding.

Table 2 shows the characteristics of the patients with intraperitoneal or intratumoral bleeding. Initiation doses of lenvatinib among them were 12,8 and $4 \mathrm{mg} /$ day in 1, 3 and 1 patient, respectively. They discontinued treatment because of AEs: case 1, liver dysfunction of grade 3; case 2, appetite loss; case 3, HCC rupture and intraperitoneal and intratumoral hemorrhage; case 4, intratumoral hemorrhage; and case 5 , leg bone fracture caused by a bone metastasis. The average dosing period was $4.4 \pm 2.2$ days. Contrast-enhanced CT (CECT) was performed within 20 days of lenvatinib commencement (median 3 days) for all five cases. In case 1 without a large tumor, HCC rupture and intraperitoneal bleeding occurred 2 weeks after discontinuation of lenvatinib. On the other hand, in four cases with large tumors, intraperitoneal or intratumoral bleeding was evident within 2-11 days (case 2, 11 days; case 3, 2 days; case 4, 3 days and case 5, 2 days) after the start of lenvatinib, although most tumor blood flow was markedly suppressed (shown in Fig. 1). Three patients had symptoms associated with hemorrhage (hypotension and bradycardia in case 3, and abdominal pain in 
case 1and 4), and the others had no symptoms. Since the liver functional reserve was reduced to Child-Pugh B status after AE development, further lenvatinib therapy was terminated. Emergency transarterial embolization (TAE) was performed to establish hemostasis in cases 1, 3 and 4, and transarterial chemoembolization (TACE) as a subsequent therapy was performed in case 5 .

Table 3 shows the comparison of patient characteristics with and without intraperitoneal or intratumoral bleeding. The patients with bleeding had larger tumors (maximum tumor size; $97.5 \pm 46.4$ $\mathrm{mm}$ and $38.2 \pm 28.8 \mathrm{~mm}$, respectively. $\mathrm{p}=0.009)$ than the patients without bleeding. The dosing period of lenvatinib (median, 3 and 93 days, respectively. $\mathrm{p}<0.001$ ) and the survival time from initial administration of lenvatinib (median, 77 and 495 days, respectively. $\mathrm{p}<0.001$ ) of the patients with bleeding were shorter than those of the patients without bleeding.

\section{Discussion/Conclusion}

Lenvatinib has recently been approved as systemic therapy for patients with advanced HCCs or earlier stage tumors progressing upon or unsuitable for loco-regional therapies. The REFLECT trial reported that lenvatinib was safe and not inferior to sorafenib in terms of overall survival, progression-free survival, and time to progression [5]. In addition, some recent real-world, retrospective, multicenter studies reported that lenvatinib exhibited high efficacy and tolerability in patients who did and did not meet the inclusion criteria of the REFLECT trial $[6,7]$.

Here, we describe the clinical courses of 68 patients with unresectable HCC who received lenvatinib. Among them, 5 cases $(7.4 \%)$ developed tumor hemorrhages, accompanied by a reduction of the liver functional reserve. The average dosing period of Lenvatinib was only $4.4 \pm 2.2$ days. Especially, 4 cases who recieved lenvatinib for large HCCs (maximum tumor diameter was $>90 \mathrm{~mm}$ ) developed tumor-related hemorrhage within 2-11 days. This study included 12 patients with large HCCs. Among them, 4 cases developed tumor hemorrhage and all 4 cases had been administered lenvatinib as the initial treatment for the primary HCCs. Such patients with large HCCs have poor prognoses, and the functional liver reserve tends to deteriorate if AEs develop, rendering further therapies impractical. Clinicians must anticipate AEs and plan appropriate treatments.

There are some other reports about hemorrhagic events in HCC patients treated with MTAs. However, the major hemorrhagic events during MTAs treatment are gastrointestinal hemorrhage, including variceal hemorrhage and intracranial hemorrhage [10,11]. Thus, to the best of our knowledge, no previous report on HCC has identified tumor-related hemorrhage as an MTA-related $\mathrm{AE}$ other than our case reports [8]. In patients with advanced thyroid cancer, fistulation and tumorrelated bleeding are well-described life-threatening AEs triggered by tumor shrinkage induced by 
lenvatinib $[12,13]$. However, in our cases, intraperitoneal or intratumoral hemorrhage developed within a few days after lenvatinib commencement, and we observed no shrinkage of the tumors.

Follow-up angiography after intratumoral hemorrhage showed contrast material pooling, consistent with the bleeding point on the $\mathrm{CT}$ in Case 4 (Figure 1h). This contrast material pooling was similar to the vascular lakes occasionally observed during TACE. In this setting, localized pooling of contrast medium is occasionally observed, resembling tumor extravasation. This angiographic finding is termed the "vascular lake phenomenon (VLP)" or the "pooling phenomenon" [14-16]. In the context of VLP caused by TACE, some authors have suggested that rapid blocking of the HCC blood flow may increase pressure within the fragile tumor microvasculature, triggering vessel rupture and blood leakage into the tumor [16]. The VLP of TACE is specific for large encapsulated tumors, and additional tumor embolization is performed until the pooling disappears to avoid VLP-associated complications including tumor rupture $[15,16]$. Our cases had huge encapsulated HCCs, and CECT revealed that the tumor blood supply was markedly suppressed. Therefore, we speculate that lenvatinib triggers something similar to the VLP induced by TACE.

The limitations of this study include its retrospective nature and the small number of patients. In addition, we are unable to explain the exact mechanism of bleeding.

In conclusion, we showed that lenvatinib induced tumor-related hemorrhage in patients with large HCCs, although most tumoral blood flow was markedly suppressed. We speculate that lenvatinib quickly blocks the feeding circulation resulting in the tumor hemorrhage after necrosis. Clinicians should pay careful attention to life-threatening hemorrhage when lenvatinib is used to treat large HCCs. 


\section{Statements}

\section{Statement of Ethics}

This study was conducted according to the guidelines of the Declaration of Helsinki. Written informed consent was obtained from all patients prior to treatment. The study protocol was approved by the Ethics Committee of Osaka City University Hospital (No. 3720).

\section{Conflict of Interest Statement}

Norifumi Kawada received research funding from Eisai Pharmaceutical Co., Ltd.

\section{Funding Sources}

The authors have no funding sources.

\section{Author Contributions}

Uchida-Kobayashi S: Data analysis and drafting the manuscript. Kageyama K and Yamamoto A:

Radiological assessment. Ikenaga H, Yoshida K, Kotani K, Kimura K amd Odagiri N: Laboratory data acquisition and Clinical data analysis. Hagihara A, Fujii H, Enomoto M and Tamori A: Hepatological and oncological assessment. Kubo S, Miki and Kawada N : Grand design of the study and drafting the manuscript. 


\section{References}

1. McGlynn KA, Petrick JL, El-Serag HB. Epidemiology of Hepatocellular Carcinoma.Hepatology. 2020 Apr 22. doi: 10.1002/hep.31288. [Epub ahead of print]

2. Bray F, Ferlay J, Soerjomataram I, Siegel RL, Torre LA, Jemal A. Global cancer statistics 2018:GLOBOCAN estimates of incidence and mortality worldwide for 36 cancers in 185 countries. CACancer J Clin 2018;68:394-424.

3. Petrick JL, Florio AA, Znaor A, Ruggieri D, Laversanne M, Alvarez CS, Ferlay J, et al. International trends in hepatocellular carcinoma incidence, 1978-2012. Int J Cancer 2019;E-pub (Oct10).

4. EASL Clinical Practice Guidelines. Management of hepatocellular carcinoma. Journal of hepatology 2018; 69: 182-236.

5. Kudo M, Finn RS, Qin S, Han K, Ikeda K, Piscaglie F, et al. Lenvatinib versus sorafenib in firstline treatment of patients with unresectable hepatocellular carcinoma: a randomised phase 3 noninferiority trial. Lancet. 2018; 391(10126): 1163-1173.

6. Sho T, Suda G, Ogawa K, Kimura M, Shimozaki T, Maehara O, et al. Early response and safety of lenvatinib for patients with advanced hepatocellular carcinoma in a real-world setting. JGH Open. 2019; 4(1): 54-60. doi: 10.1002/jgh3.12209. eCollection 2020 Feb.

7. Ohki T, Sato K, Kondo M, Goto E, Sato T, Kondo Y, et al. Impact of Adverse Events on the Progression-

Free Survival of Patients with Advanced Hepatocellular Carcinoma Treated with Lenvatinib: A Multicenter Retrospective Study. Drugs Real World Outcomes. 2020 Feb 11. doi: 10.1007/s40801-020-00179-7. [Epub ahead of print]

8. Kotani K, Uchida S, Yoshida K, Kawamura E, Fujii H, Hagihara A, et al. Lenvatinib-induced tumor-related hemorrhage in patients with unresectable hepatocellular carcinoma. Am J Gastroenterol. In press.

9. Bruix J, Reig M, Sherman M. Evidence-Based Diagnosis, Staging, and Treatment of Patients With Hepatocellular Carcinoma. Gastroenterology. 2016;150(4):835-853.

10. Duffy A, Wilkerson J, Greten TF. Hemorrhagic events in hepatocellular carcinoma patients treated with antiangiogenic therapies. Hepatology. 2013; 57(3): 1068-1077.

11. Li X, Wan J, Wu Z, Tu J, Hu Y, Wu S,et al.

Fatal adverse events with molecular targeted agents in the treatment of advanced hepatocellular carcinoma: a metaanalysis of randomized controlled trials. Drug Des Devel Ther. 2018;12:3043-3049.

12. Masaki C, Sugino K, Saito N, Saito Y, Tanaka T, Ogimi Y, et al. Lenvatinib induces early tumor 
shrinkage in patients with advanced thyroid carcinoma. Endocr J. 2017; 64(8): 819-826. doi: 10.1507/endocrj.EJ17-0104. Epub 2017 Jul 28.

13. Staub Y, Nishiyama A, Suga Y, Fujita M, Matsushita R, Yano S. Clinical Characteristics Associated With Lenvatinib-induced Fistula and Tumor-related Bleeding in Patients With Thyroid Cancer. Anticancer Res. 2019 ;39(7):3871-3878. doi: 10.21873/anticanres.13537.

14. Hu B, Zhong BY, Zhang L, Dai C, Li W, Shen J, et al. Occurrence of Vascular Lake Phenomenon Before Embolization for the Prediction of Lipiodol Uptake for Intermediate-Stage Hepatocellular Carcinoma Patients that Underwent cTACE. Cardiovasc Intervent Radiol. 2020. Epub ahead of print.

15. Seki A, Hori S, Shimono C. Management of vascular lake phenomenom on angiography during chemoembolization with superabsorbent polymer microspheres. Jpn J Radiol. 20 15;33(12):741748.

16. Cavalcante RN, Nasser F, Motta-Leal-Filho JM, Affonso BB, Galstri FL, Fina BD, et al. Occurrence of Vascular Lake Phenomenon as a Predictor of Improved Tumor Response in HCC Patients That Underwent DEB-TACE. Cardiovasc Intervent Radiol. 2017;40(7):10441051. 


\section{Figure Legends}

Fig. 1. Images taken before and after lenvatinib administration in patients with tumor hemorrhage. Arterial phase imaging of contrast enhanced CT before (a, c, f, i) and after (b, d, e, g, j) lenvatinib administration.

Digital subtraction angiography imaging at intratumor hemorrhage (h).

White arrows: intratumor hemorrhage with vascular lake-like phenomenon

White arrow heads: intraperitoneal hematoma 


\section{Case 2}

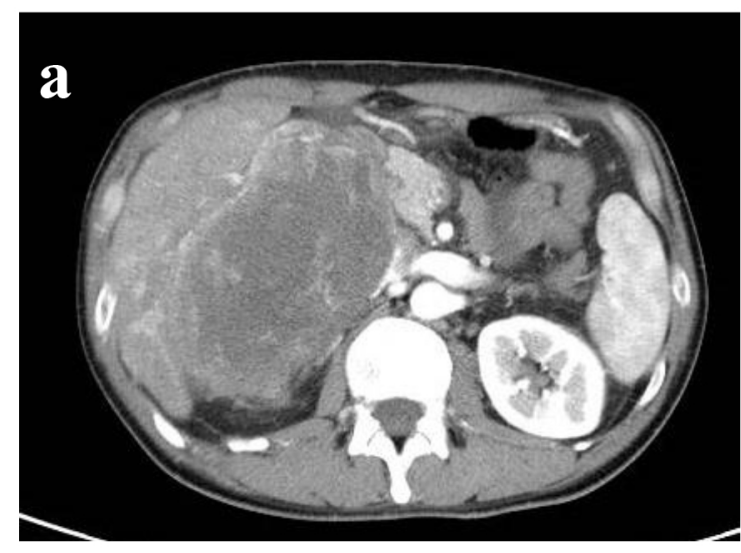

After administration of lenvatinib

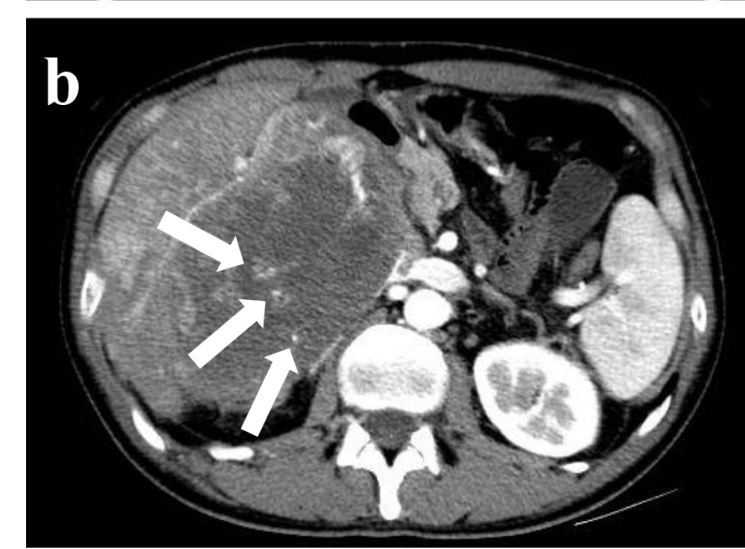

Case 3
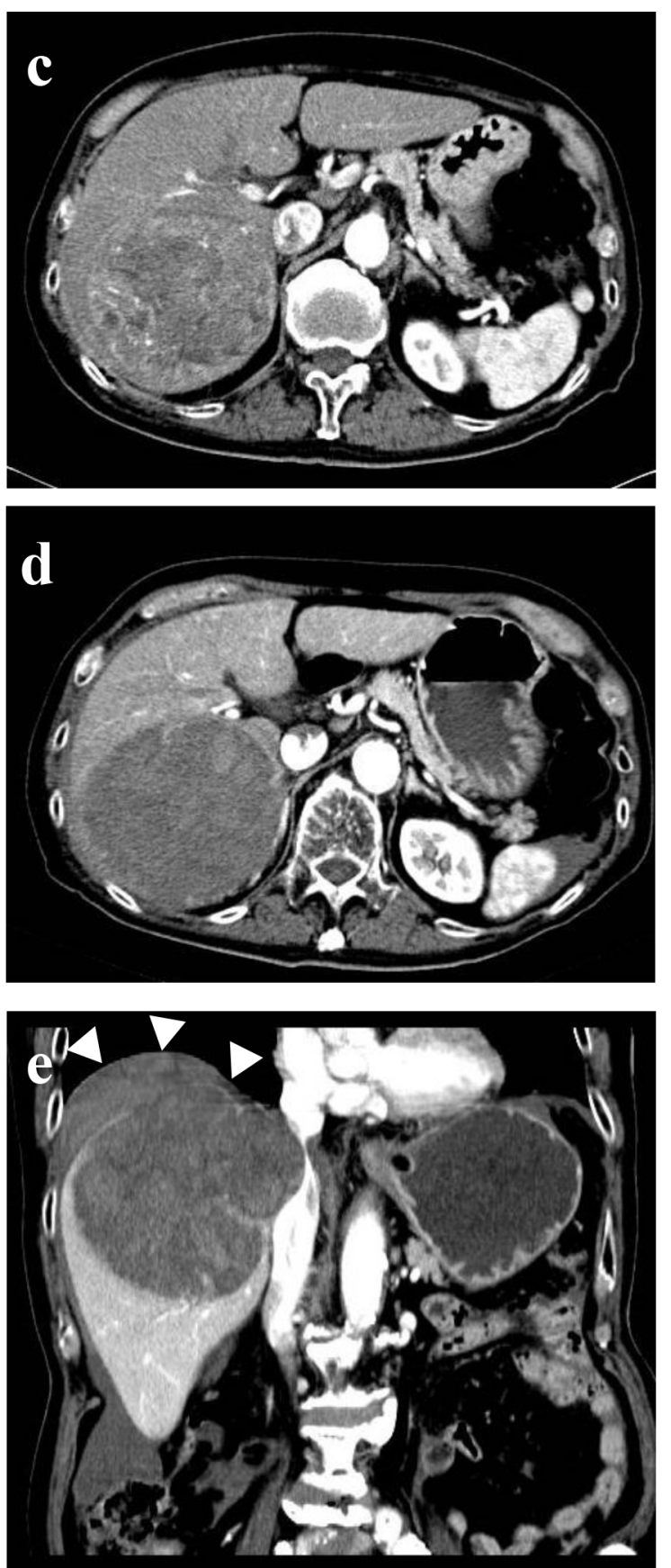

Case 4
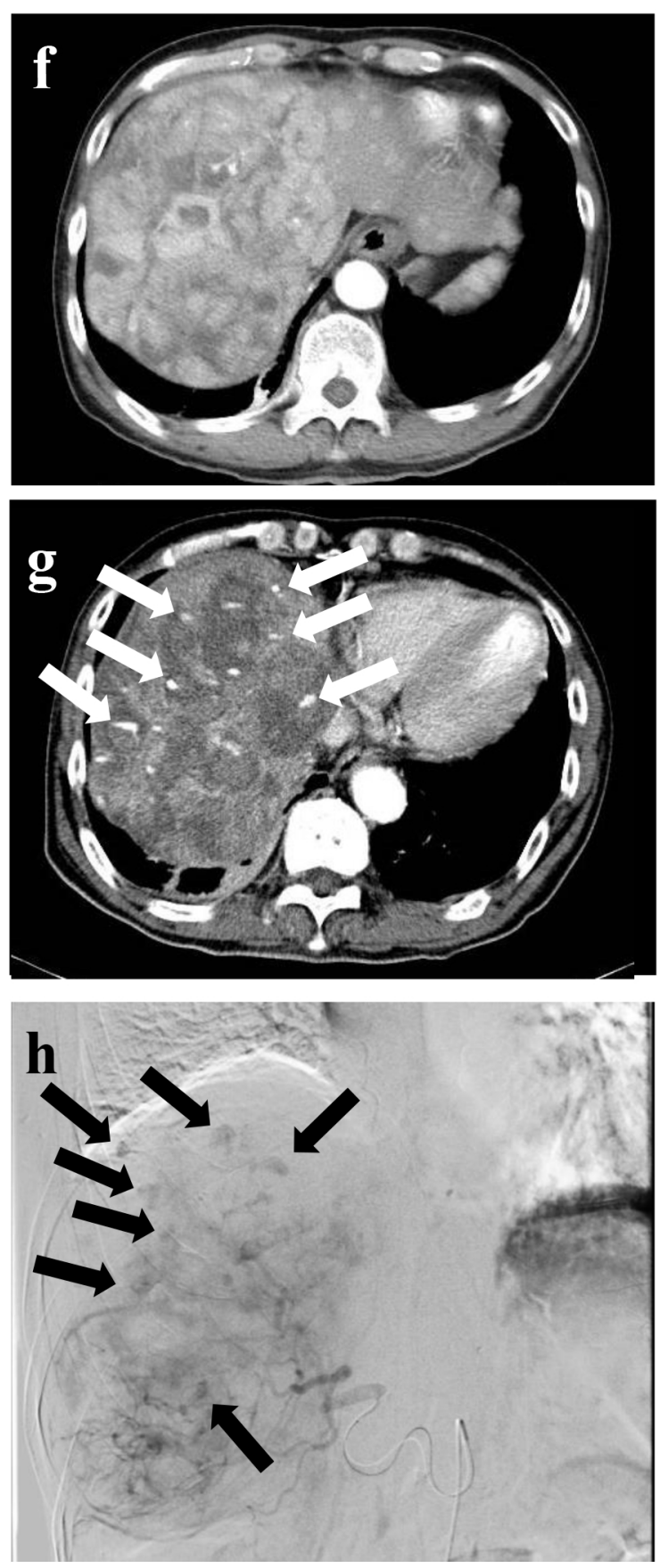

Case 5
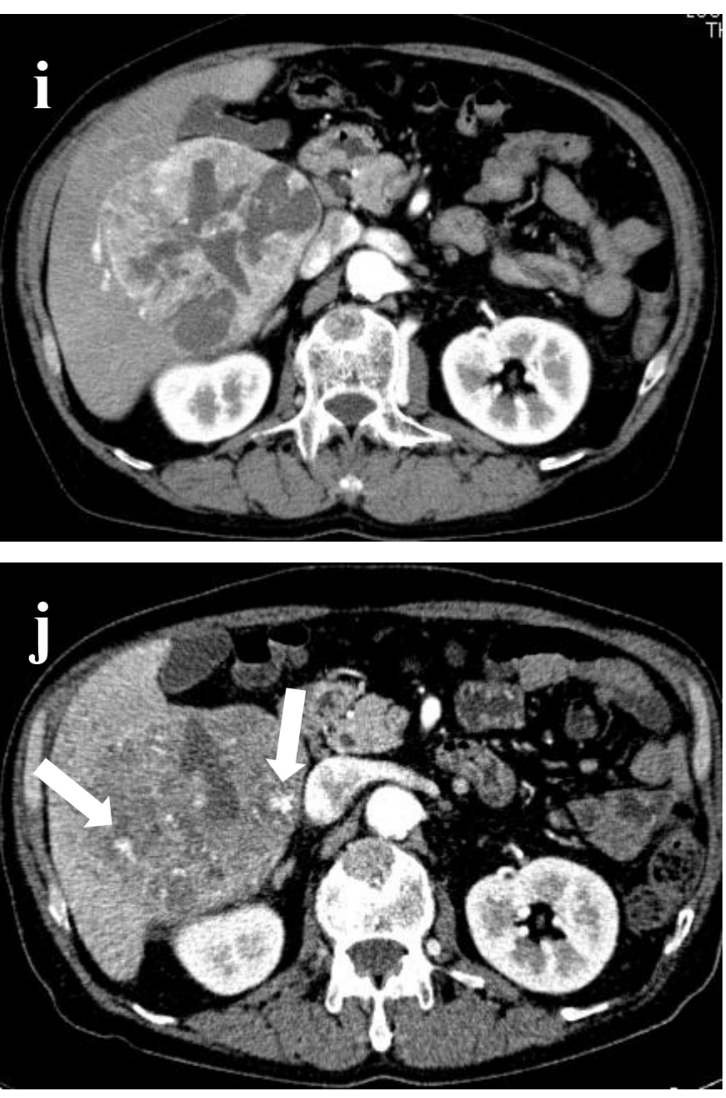

Fig. 1. 


\section{Table}

Table 1. Baseline patient characteristics

\begin{tabular}{lc}
\hline & $\mathrm{n}=68$ \\
\hline age (years) & $72(55-78)$ \\
male, $\mathrm{n}(\%)$ & $59(86.8)$ \\
HCVAb positive, n (\%) & $29(42.6)$ \\
HBsAg positive, n (\%) & $14(20.6)$ \\
Child-Pugh A, n (\%) & $63(92.7)$ \\
previous HCC treatment negative, n (\%) & $6(8.8)$ \\
Maximum tumor size (mm) & $42.6 \pm 33.7$ \\
BCLC-C, n (\%) & $26(38.2)$ \\
AFP (ng/mL) & $20.1(3.6-684.6)$ \\
PIVKA-II (mAU/mL) & $212(60-3638)$ \\
\end{tabular}

HCV Ab; hepatitis C virus antibody, HBsAg; hepatitis B surface antigen, AFP; alpha-

Fetoprotein, PIVKA-II; protein induced by vitamin K absence or antagonist II, BCLC;

Barcelona Clinic Liver Cancer 
Table 2. Baseline characteristics of the patients with intraperitoneal or intratumoral bleeding

\begin{tabular}{|c|c|c|c|c|c|c|c|c|c|c|c|c|c|}
\hline case & age & sex & HCV Ab & HBsAg & $\begin{array}{c}\text { Child-Pugh } \\
\text { score }\end{array}$ & $\begin{array}{c}\text { previous } \\
\text { HCC } \\
\text { treatment }\end{array}$ & $\begin{array}{c}\text { AFP } \\
(\mathrm{ng} / \mathrm{mL})\end{array}$ & $\begin{array}{l}\text { PIVKA-II } \\
(\mathrm{mAU} / \mathrm{mL})\end{array}$ & $\begin{array}{c}\text { maximum } \\
\text { tumor } \\
\text { size }(\mathrm{mm})\end{array}$ & $\begin{array}{c}\text { intrahepatic } \\
\text { lesion }\end{array}$ & $\begin{array}{l}\text { vascular } \\
\text { invasion }\end{array}$ & metastasis & $\begin{array}{r}\text { BCLC } \\
\text { stage }\end{array}$ \\
\hline 1 & 81 & male & + & - & 6 & + & 21934 & 98421 & 23.0 & multiple & + & - & $\mathrm{C}$ \\
\hline 2 & 45 & male & - & + & 6 & - & 21934 & 98421 & 147.6 & multiple & + & + & $\mathrm{C}$ \\
\hline 3 & 85 & male & + & - & 5 & - & 1082.2 & 8934 & 108.8 & single & - & - & A \\
\hline 4 & 63 & male & + & - & 5 & - & 81.2 & 3948 & 91.4 & multiple & - & + & $\mathrm{C}$ \\
\hline 5 & 65 & male & - & - & 5 & - & 2.0 & 44 & 116.7 & multiple & + & + & $\mathrm{C}$ \\
\hline
\end{tabular}

HCV Ab; hepatitis C virus antibody, HBsAg; hepatitis B surface antigen, HCC; hepatocellular carcinoma, AFP; alpha-Fetoprotein,

PIVKA-II; protein induced by vitamin K absence or antagonist II, BCLC; Barcelona Clinic Liver Cancer 
Table 3. Comparison of patient characteristics with and without intraperitoneal or intratumoral bleeding

\begin{tabular}{lccr}
\hline & $\begin{array}{c}\text { With bleeding } \\
(\mathrm{n}=5)\end{array}$ & $\begin{array}{c}\text { Without bleeding } \\
(\mathrm{n}=63)\end{array}$ & $\mathrm{p}$ value \\
\hline age (years) & $65(63-81)$ & $72(67-77)$ & 0.724 \\
male, $\mathrm{n}(\%)$ & $5(100.0)$ & $59(93.7)$ & 0.563 \\
HCVAb positive, $\mathrm{n}(\%)$ & $2(20.0)$ & $26(41.3)$ & 0.553 \\
HBsAg positive, $\mathrm{n}(\%)$ & $2(40.0)$ & $13(20.6)$ & 0.545 \\
Child-Pugh A, $\mathrm{n}(\%)$ & $5(100.0)$ & $58(92.1)$ & 0.514 \\
previous HCC treatment & $4(80.0)$ & $2(3.1)$ & 0.075 \\
negative, $\mathrm{n}(\%)$ & $97.5 \pm 46.4$ & $38.2 \pm 28.8$ & 0.009 \\
Maximum tumor size (mm) & $3(60.0)$ & $23(36.5)$ & 0.433 \\
BCLC-C, $\mathrm{n}(\%)$ & $85.6(81.2-1417.1)$ & $15.7(3.6-569.9)$ & 0.359 \\
AFP & $3948(2987-8364)$ & $168(60-3000)$ & 0.113 \\
PIVKA-II & $3(3-5)$ & $93(40-147)$ & $<0.001$ \\
Median dosing period of LEN & & & $<0.001$ \\
(days) & $77(46-108)$ & $495(323-667)$ & \\
Median survival time from initial & & & \\
administration of LEN (days) & &
\end{tabular}

HCV Ab; hepatitis C virus antibody, HBsAg; hepatitis B surface antigen, HCC; hepatocellular carcinoma, BCLC; Barcelona Clinic Liver Cancer, AFP; alphaFetoprotein, PIVKA-II; protein induced by vitamin K absence or antagonist II, LEN; lenvatinib 